\title{
Gut microbiome associations with breast cancer risk factors and tumor characteristics: a pilot study
}

\author{
Anna H. Wu ${ }^{1}$. Chiuchen Tseng ${ }^{1} \cdot$ Cheryl Vigen ${ }^{2} \cdot$ Yang Yu $^{3} \cdot$ Wendy Cozen $^{1} \cdot$ Agustin A. Garcia ${ }^{4} \cdot$ Darcy Spicer $^{5}$
}

Received: 21 November 2019 / Accepted: 21 May 2020 / Published online: 28 May 2020

(c) The Author(s) 2020

\begin{abstract}
Objective To investigate the association between gut microbiome with breast tumor characteristics (receptor status, stage and grade) and known breast cancer risk factors.

Methods In a pilot cross-sectional study of 37 incident breast cancer patients, fecal samples collected prior to chemotherapy were analyzed by $16 \mathrm{~S}$ ribosomal RNA (rRNA) gene-based sequencing protocol. Alpha diversity and specific taxa by tumor characteristics and breast cancer risk factors were tested by Wilcoxon rank sum test, and by differential abundance analysis, using a zero-inflated negative binomial regression model with adjustment for total counts, age and race/ethnicity.

Results There were no significant alpha diversity or phyla differences by estrogen/progesterone receptor status, tumor grade, stage, parity and body mass index. However, women with human epidermal growth factor receptor 2 positive (HER2+) $(n=12)$ compared to HER2 - $(n=25)$ breast cancer showed $12-23 \%$ lower alpha diversity [number of species (OTU) $p=0.033$, Shannon index $p=0.034]$, lower abundance of Firmicutes $(p=0.005)$ and higher abundance of Bacteroidetes $(p=0.089)$. Early menarche (ages $\leq 11)(n=11)$ compared with later menarche (ages $\geq 12)(n=26)$ was associated with lower OTU $(p=0.036)$, Chaol index $(p=0.020)$ and lower abundance of Firmicutes $(p=0.048)$. High total body fat (TBF) $(>46 \%)(n=12)$ compared to lower $(\leq 46 \%)$ TBF was also associated with lower Chao 1 index $(p=0.011)$. There were other significant taxa abundance differences by HER2 status, menarche age, as well as other tumor and breast cancer risk factors. Conclusions and relevance Further studies are needed to identify characteristics of the human microbiome and the interrelationships between breast cancer hormone receptor status and established breast cancer risk factors.
\end{abstract}

Keywords Microbiome $\cdot$ Tumor characteristics $\cdot$ HER2 status $\cdot$ Age at menarche

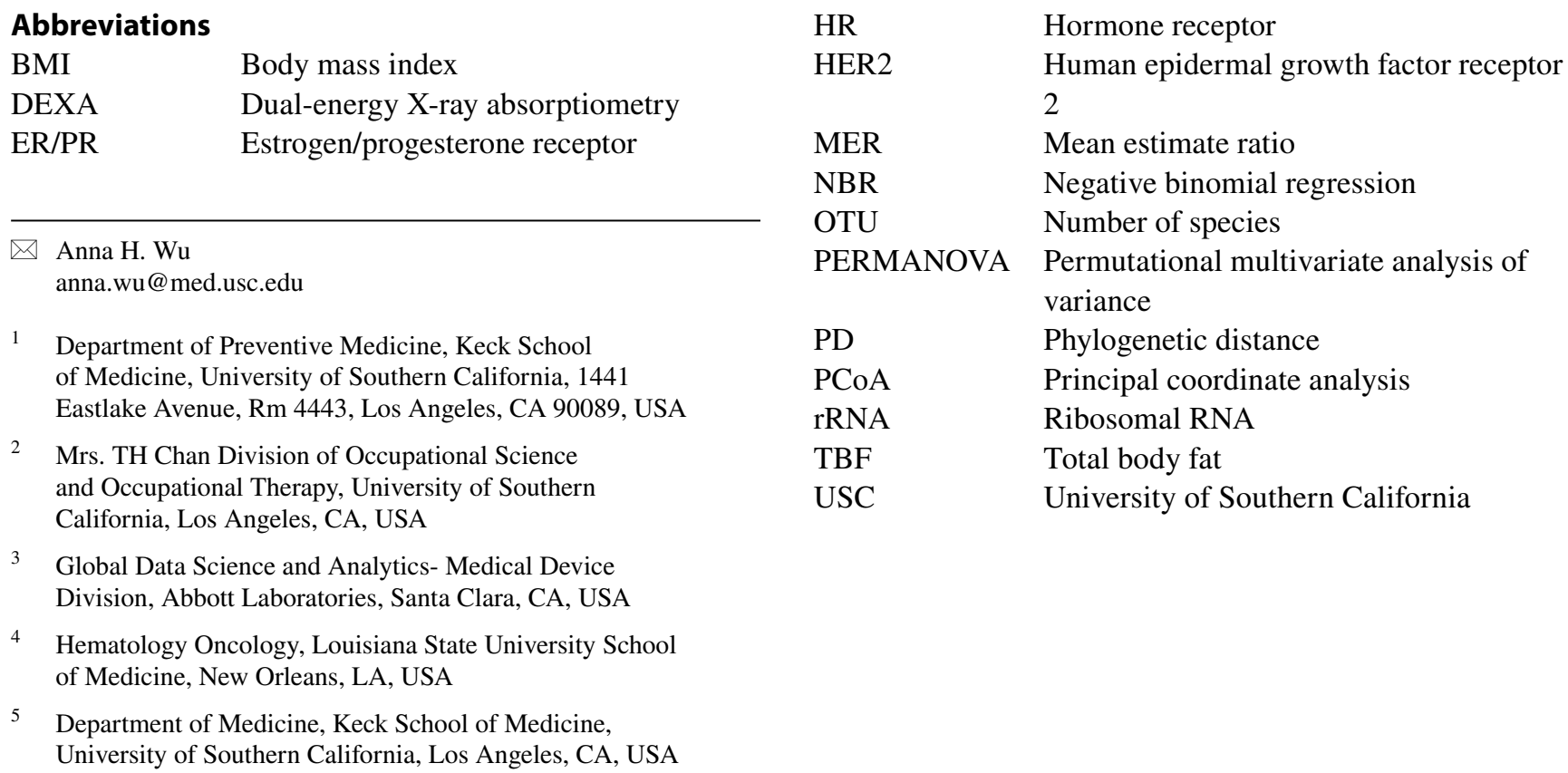




\section{Background}

In the past decade numerous intriguing links between the gut microbiota and risk of obesity, metabolic diseases and inflammatory responses have been reported $[1,2]$ but less is known about the gut microbiota of breast cancer patients [3, 4]. A study conducted in Kaiser Permanente health care members of pretreatment samples showed that after adjusting for age, body mass index (BMI), and other factors, postmenopausal women diagnosed with incident breast cancer $(n=48)$ compared to control women $(n=48)$ showed significantly lower alpha diversity in fecal microbiota, and differing relative abundance of select taxa of Firmicutes (Clostridiaceae, Faecalibacterium, Ruminococcaceae, Dorea and Lachnospiraceae) [5]. Low gut microbial diversity has been associated with obesity, insulin resistance, and other factors some of which are aligned to risk of breast cancer [6]. In a case-only study of 31 women diagnosed with early stage breast cancer [7], the total number of unique species of Bacteroidetes, and Firmicutes differed significantly by tumor stage and abundance of Firmicutes was 16\% lower among those with overweight BMI $\left(\geq 25 \mathrm{~kg} / \mathrm{m}^{2}\right)$ than those with normal BMI $(p=0.06)$.

Breast cancer is a heterogenous disease with multiple subtypes that display distinct risk factor patterns with differences between estrogen receptor (ER)/progesterone receptor $(\mathrm{PR})$ positive $(\mathrm{ER}+\mathrm{PR}+)$ versus those that are negative for ER/PR [8-10]. Breast cancers that are positive for human epidermal growth factor (HER2+) also differ from those that are HER2-, and triple negative (ER-PR-HER2-) breast cancers are the most deadly $[9,11]$. It is not known whether different breast cancer subtypes are associated with distinct microbial signatures. Several studies have also explored the role of breast tissue microbiome in modulating the risk of breast cancer [12-17]. We are aware of one study that applied a panpathogen microarray (PathoChip) strategy on formalin fixed paraffin embedded samples of breast tissues to investigate microbial patterns by different breast cancer subtypes, but this study lacked information on tumor stage or grade or breast cancer risk factors [18].

We describe below results from a cross-sectional analysis conducted among 37 women diagnosed with incident breast cancer in Los Angeles County to further investigate whether gut microbiome prior to breast cancer chemotherapy differs by receptor status (ER, PR, HER2) and stage and grade of breast cancer. We also investigated whether gut microbiome profile differed by well-established breast cancer risk factors including age at menarche, parity, baseline BMI, and physical activity.

\section{Materials and methods}

\section{Patient population and specimen collection}

This study was conducted at the University of Southern California (USC) Norris Comprehensive Cancer Center and at the Los Angeles County + USC Medical Center. Women of all race/ethnicities, newly diagnosed with incident invasive breast cancer were considered potentially eligible. Exclusionary criteria included recurrent breast cancer, a history of other cancers (other than non-melanoma skin cancer), celiac disease, inflammatory bowel disease, bariatric surgery, pregnancy or nursing within past 12 months, past treatment with chemotherapy, antibiotic use (defined as 1 week or more during the month prior to baseline fecal sample collection), or use of probiotic supplements or prednisone. After signing informed consent, eligible and willing patients donated up to four fecal specimens and completed up to four clinical visits during an average of 9 months follow-up. Baseline specimens were collected before chemotherapy started for those who received neoadjuvant chemotherapy and were collected after surgery but before chemotherapy for those who received adjuvant chemotherapy or only had surgery (Fig. 1). The study protocol was approved by the USC Institutional Review Board.
Fig. 1 Collection of baseline (B) and last (L) fecal samples from study participants

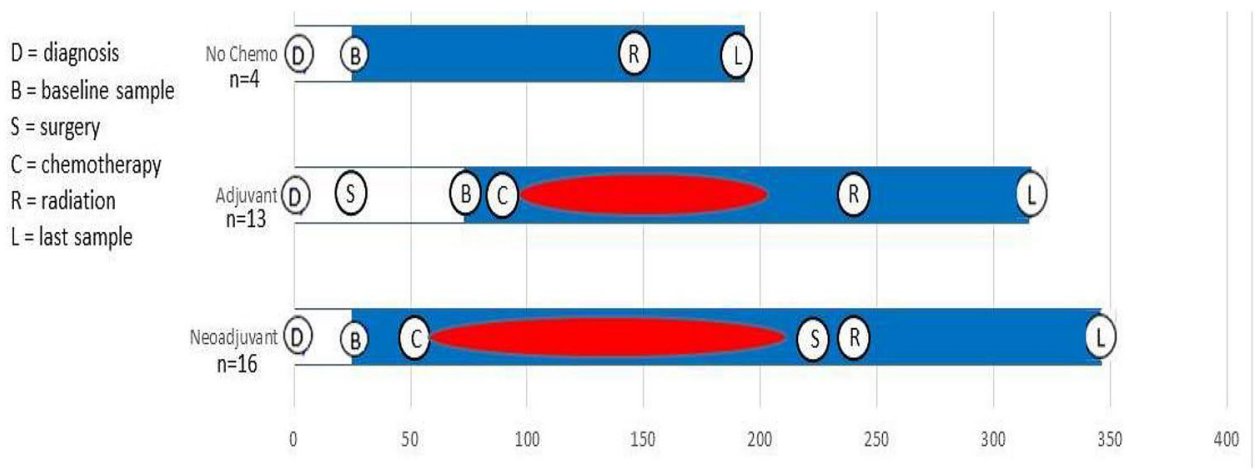


We used a fecal specimen collection kit with illustrated instructions that was designed and tested at the University of Maryland [19]. Participants were given collection kits and obtained samples using the provided pre-labeled collection devices and tubes containing the nucleic acid preservative RNAlater. All fecal samples were discreetly stored in the participants' home freezers, and were either picked up by the study staff or brought in to USC by the study participants. These stool samples were then stored in the $-80{ }^{\circ} \mathrm{C}$ freezers of Preventive Medicine laboratory at USC until they were sent for measurement at the completion of the study. Body composition data obtained from the dual-energy $\mathrm{x}$-ray absorptiometry (DEXA) scans at the first clinic visit (baseline) were included in our analysis. The DEXA scan was conducted at the USC Integrative Center for Oncology Research in Exercise. Participants also completed a baseline questionnaire to assess menstrual and reproductive history, medical history (e.g., hypertension, diabetes, benign breast diseases), family history of cancer, use of medications, and other lifestyle factors. Only the baseline fecal sample, i.e., collected before chemotherapy was included in the data analysis of this paper. Fecal samples collected during and after completion of breast cancer treatment are still under investigation.

\section{Fecal specimen processing and microbiome analyses}

Microbiome analyses were conducted in the laboratory of Dr. Jacques Ravel using his well-established methods, including DNA extraction, 16S rRNA gene amplification of the two barcoded universal primers $319 \mathrm{~F}$ and $806 \mathrm{R}$ for PCR amplification of the V3 and V4 hypervariable regions and sequencing the amplicons on the Illumina MiSeq platform $[5,19]$. The $16 \mathrm{~S}$ rRNA genes were amplified in 96-well microtiter plates. Negative controls without a template were processed for each primer pair. They performed taxonomic assignments and generated taxa abundance and read count tables for each of the 144 fecal samples we collected from 38 breast cancer patients. After we excluded 14 samples with low $(<100)$ read counts (referred to as failed), 130 samples remained from 37 patients as all 4 samples failed in one patient and she was excluded from all subsequent analyses. Hence this current analysis is comprised of baseline samples from 37 women diagnosed with incident breast cancer (Table 1).

\section{Statistical analyses}

Microbiome alpha diversity was estimated after rarefaction using four measures: (a) counts of observed species (OTUs) unadjusted for relative abundances; (b) Chao1 as an estimate of the species richness; (c) Shannon index to measure both richness and evenness, and (d) phylogenetic distance (PD whole tree) in the diversity calculation. We used Wilcoxon rank sum test to examine differences in the alpha diversity between any two groups of interest (e.g., HER2+ vs HER2-) and Kruskal-Wallis to examine differences between any three groups of interest (e.g., age at menarche $\leq 11,12, \geq 13$ ).

We conducted permutational multivariate analysis of variance (PERMANOVA) to test statistical significance of overall composition and to examine the relationship with personal factors including age $(<50,50+)$, race (Hispanic, not Hispanic), menopausal status (pre- menopause, postmenopause); age at menarche $(\leq 11, \geq 12), \operatorname{BMI}(<25, \geq 25)$, total body fat $(\mathrm{TBF})(\leq 46 \%,>46 \%)$, parity (nulliparous, parous), physical activity (no, yes), and tumor characteristics including stage(I/II, III), grade (I/II, III); receptor status (ER/PR: ER+PR+, ER+PR-, ER-PR-) and HER2 status (HER2-, HER2+).

The relationship of overall gut microbiome composition with personal factors (age, menopause status, race/ethnicity, age at menarche, parity, physical activity, BMI, TBF) and tumor characteristics was assessed by principal coordinate analysis (PCoA) based on the unweighted (qualitative) UniFrac distance matrix [20]. PCoA plots were generated using the first two principal coordinates, according to categories of personal and tumor characteristics.

Turning to taxonomy, we investigated the 201 specific genera that were present in at least $25 \%$ of our study samples. To accommodate the sparse, non-normally distributed count data, we conducted differential abundance analysis, using a zero-inflated negative binomial regression (NBR) model [21] provided by SAS proc genmod, to examine relationships of specific taxa to tumor characteristics and breast cancer risk factors. We investigated differences in taxa between groups with adjustment for total counts (Model 1), as well as age $(<49,50-59,60+)$ and race/ethnicity (Hispanic vs non-Hispanic) (Model 2). The presumed lower risk categories [e.g., HER-, ER+, $\mathrm{PR}+$, lower stage $(0 / \mathrm{I})$, lower grade (I/II), later age at menarche ( $\geq 12$ years), parous, physically active, lower BMI $\left(<25 \mathrm{~kg} / \mathrm{m}^{2}\right)$, and lower TBF $\left.(\leq 46 \%)\right]$ were used as the reference groups in the NBR analysis. The mean estimate ratio (MER) under the NBR model represents the ratio of the log estimate in one group versus the reference group and the $p$ value is the probability of obtaining such a ratio under the null hypothesis. Thus, if the mean abundance of a taxon is higher in the HER2+ than in the HER2 - group (reference group), we expect a MER greater than one. On the other hand, if the mean abundance of a taxon is lower among HER2+ than HER2- tumors, we expect a MER less than one. A probability of $P \leq 0.001$ was accepted as significant in this study. Results were 
Table 1 Characteristics of 37 breast cancer patients by human epidermal growth factor receptor 2 (HER2) status $[N(\%)$ or $\mathrm{M} \pm \mathrm{SD}]$

\begin{tabular}{|c|c|c|c|c|}
\hline & \multirow[t]{2}{*}{ All } & \multicolumn{2}{|l|}{ HER2 status } & \multirow[t]{2}{*}{$p$ value } \\
\hline & & Negative & Positive & \\
\hline$N$ & 37 & 25 & 12 & \\
\hline Mean age $\pm \mathrm{SD}$ & $50.6 \pm 12.3$ & $51.7 \pm 13.7$ & $48.3 \pm 8.93$ & $0.43^{\mathrm{a}}$ \\
\hline \multicolumn{5}{|l|}{ Menopausal status } \\
\hline Premenopause & $20(54)$ & $12(48)$ & $8(67)$ & \\
\hline Postmenopause & $17(46)$ & $13(52)$ & $4(33)$ & $0.32^{\mathrm{b}}$ \\
\hline \multicolumn{5}{|l|}{ Race/ethnicity } \\
\hline Hispanic & $27(73)$ & $18(72)$ & $9(75)$ & \\
\hline Non-Hispanic & $10(27)$ & $7(28)$ & $3(25)$ & $1.00^{\mathrm{b}}$ \\
\hline \multicolumn{5}{|c|}{ Body mass index (BMI), kg/m² } \\
\hline Mean $\mathrm{BMI} \pm \mathrm{SD}$ & $30.6 \pm 7.9$ & $31.2 \pm 8.3$ & $29.5 \pm 7.1$ & $0.67^{\mathrm{a}}$ \\
\hline$<25$ & $9(24)$ & $7(28)$ & $2(17)$ & \\
\hline $25-30$ & $14(38)$ & $8(32)$ & $6(50)$ & \\
\hline$>30$ & $14(38)$ & $10(40)$ & $4(33)$ & $0.67^{\mathrm{b}}$ \\
\hline \multicolumn{5}{|l|}{ Total body fat (TBF) } \\
\hline Mean $\%$ fat \pm SD & $42.7 \pm 6.9$ & $42.6 \pm 7.5$ & $42.9 \pm 5.8$ & $0.90^{\mathrm{a}}$ \\
\hline$\leq 46 \%$ & $25(68)$ & $19(76)$ & $6(50)$ & \\
\hline$>46 \%$ & $12(32)$ & $6(24)$ & $6(50)$ & $0.15^{\mathrm{b}}$ \\
\hline \multicolumn{5}{|l|}{$\mathrm{BMI}$ and $\mathrm{TBF}$} \\
\hline I $(<25 \& \leq 46 \%)$ & $9(24)$ & $7(28)$ & $2(17)$ & \\
\hline II $(\geq 25 \& \leq 46 \%)$ & $16(43)$ & $12(48)$ & $4(33)$ & \\
\hline III $(\geq 25 \&>46 \%)$ & $12(32)$ & $6(24)$ & $6(50)$ & $0.36^{\mathrm{b}}$ \\
\hline \multicolumn{5}{|l|}{ Age at menarche } \\
\hline Mean age \pm SD & $12.4 \pm 1.5$ & $12.2 \pm 1.5$ & $12.7 \pm 1.5$ & $0.49^{\mathrm{a}}$ \\
\hline$\leq 11$ & $11(30)$ & $7(28)$ & $4(33)$ & \\
\hline 12 & $9(24)$ & $7(28)$ & $2(17)$ & \\
\hline$\geq 13$ & $17(46)$ & $11(44)$ & $6(50)$ & $0.81^{b}$ \\
\hline \multicolumn{5}{|l|}{ Parity } \\
\hline Mean parity $\pm S D$ & $1.8 \pm 1.3$ & $1.8 \pm 1.2$ & $1.9 \pm 1.6$ & $0.73^{\mathrm{a}}$ \\
\hline No & $8(22)$ & $6(24)$ & $2(17)$ & \\
\hline $1-2$ & $18(49)$ & $13(52)$ & $5(42)$ & \\
\hline$\geq 3$ & $11(30)$ & $6(24)$ & $5(42)$ & $0.65^{b}$ \\
\hline \multicolumn{5}{|l|}{ Stage at diagnosis } \\
\hline $\mathrm{I} / \mathrm{II}$ & $22(59)$ & $15(60)$ & $7(58)$ & \\
\hline III & $15(41)$ & $10(40)$ & $5(42)$ & $1.00^{\mathrm{b}}$ \\
\hline \multicolumn{5}{|l|}{ Grade of tumor } \\
\hline $\mathrm{I} / \mathrm{II}$ & $14(38)$ & $12(48)$ & $2(17)$ & $0.08^{\mathrm{b}}$ \\
\hline III & $23(62)$ & $13(52)$ & $10(83)$ & \\
\hline \multicolumn{5}{|l|}{ ER/PR status } \\
\hline $\mathrm{ER}+\mathrm{PR}+$ & $23(62)$ & $19(76)$ & $4(33)$ & \\
\hline $\mathrm{ER}+\mathrm{PR}-$ & $5(14)$ & $0(0)$ & $5(42)$ & \\
\hline ER-PR- & $9(24)$ & $6(24)$ & $3(25)$ & $0.001^{\mathrm{b}}$ \\
\hline
\end{tabular}

${ }^{a}$ Wilcoxon rank sum test between HER2+ vs HER2 - group for age, BMI, parity, and age at menarche

${ }^{\mathrm{b}}$ Fisher exact test between HER2+ vs HER2- group for all other variables similar for Model 1 and 2 and we showed statistically significant MERs in NBR from Model 2 (Tables 3, 4, 5 and 6). For this pilot study we did not adjust for multiple testing [22]. All data were analyzed using R (R Foundation for Statistical Computing Vienna, Austria or SAS version 9.4 (SAS, Cary, NC). 


\section{Results}

The 37 breast cancer patients had an average age of $50.6 \pm 12.3,73 \%$ were Hispanic $(n=27), 54 \%$ were premenopausal $(n=20), 21 \%(n=8)$ were nulliparous, mean age of menarche of $12.4 \pm 1.5$, and baseline BMI of $30.6 \pm 7.9 \mathrm{~kg} / \mathrm{m}^{2}$ and TBF of $42.7 \% \pm 6.9$. Most had early stage (I/II) $(n=22,59.5 \%)$, high grade (III) $(n=23,62.2 \%)$, hormone receptor positive $(\mathrm{ER}+\mathrm{PR}+)(n=23,62.2 \%)$, and HER2 - breast cancer $(n=25,67.6 \%)$ (Table 1$)$. Women with HER2+ breast cancer were more likely to have PR- breast cancer; $66.7 \%$ of patients with HER $2+$ breast cancer had PR- breast cancer compared to $24 \%$ of those with HER2- breast cancer $(p=0.001)$.

\section{PERMANOVA analysis of personal and tumor characteristics with the unweighted UniFrac distance matrix}

Beta diversity (between-subjects species diversity) was assessed using the unweighted and weighted UniFrac distance. BMI was associated with baseline gut microbiome composition. Axis 1 explained $20.9 \%$ of all variance while axis 2 explained $10.5 \%$ (Fig. 2). Separation between the baseline microbiota of the BMI groups ( $<25 \mathrm{vs} \geq 25 \mathrm{~kg}$ / $\left.\mathrm{m}^{2}\right)$ differed for axis $1(p=0.20)$ and axis $2(p=0.024)$ with the unweighted UniFrac distance matrix but not with the weighted UniFrac distance (Fig. 2). Separation of baseline

Axis 1: $p(1 d f)=0.20$, Axis $2: p(1 d f)=0.024$

(A)

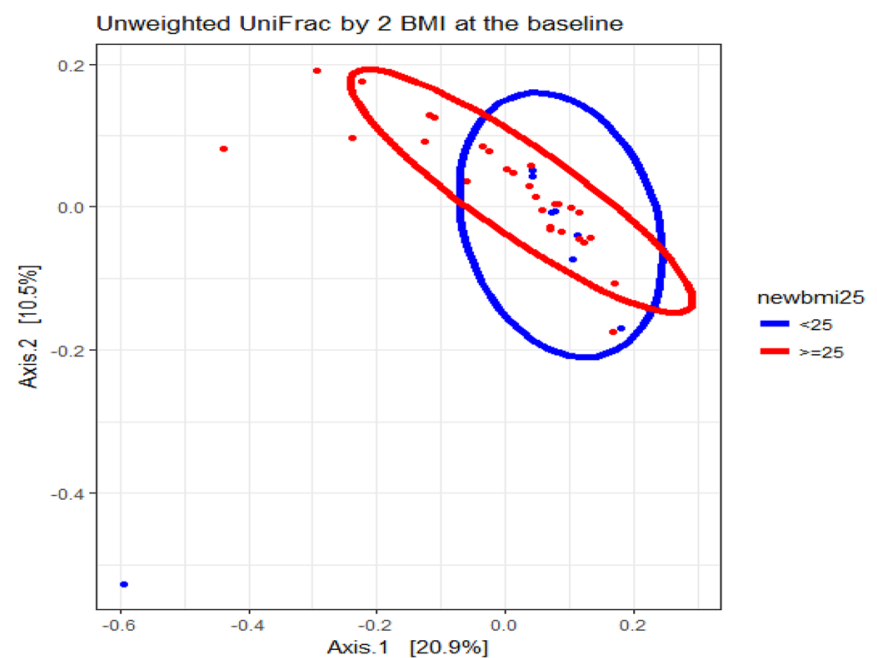

Fig. 2 Beta-diversity results by baseline body mass index are shown: A unweighted UniFrac-based principal component analysis plot of the first two principal coordinates categorized by body mass index $\left(\mathrm{BMI}<25 \mathrm{~kg} / \mathrm{m}^{2} n=9, \mathrm{BMI} \geq 25 \mathrm{~kg} / \mathrm{m}^{2} n=28\right)$. Axis 1 explained microbiota was also observed using cutpoints of $<30$ vs $\geq 30$ for BMI (axis $1 p=0.16$; axis $2 p=0.009$ ) and $<46 \%$ vs $\geq 46 \%$ for TBF (axis $1 p=0.21$; axis $2 p=0.048$ ). None of the other factors were associated with overall fecal composition (data not shown).

\section{Alpha diversity by tumor characteristics and personal characteristics}

There were no statistically significant baseline alpha diversity (within-subject species diversity) differences by tumor stage and grade, ER or PR status (Table 2). However, alpha diversity measures were $12 \%$ to $23 \%$ lower for HER $2+(n=12)$ than HER $2-(n=25)$ breast cancer; including lower OTU ( $p=0.033)$, Chao1 index $(p=0.073)$, and Shannon index $(p=0.035)$. High $(>46 \%)$ TBF compared to lower $(\leq 46 \%) \mathrm{TBF}$ was associated with lower Chao 1 index $(p=0.011)$ and OTU $(p=0.059)$. Similar patterns of differences were observed for those with normal BMI versus overweight or obese. Alpha diversity measures were lower among women with early $(\leq 11)$ than later $(\geq 12)$ age of menarche; these differences were statistically significant for OTU $(p=0.034)$, Chao 1 index $(p=0.020)$ and borderline statistically significant for Shannon index $(p=0.057)$ and PD whole tree $(p=0.073)$. Those who were physically active had higher Chao 1 index $(p=0.07)$ and OTU $(p=0.58)$ than those who were not physically active but Shannon index and PD tree were not higher. Alpha diversity measures did not differ between parous and nulliparous women.

Axis 1: $p(1 d f)=0.68$, Axis 2: $p(1 d f)=0.97$

(B)

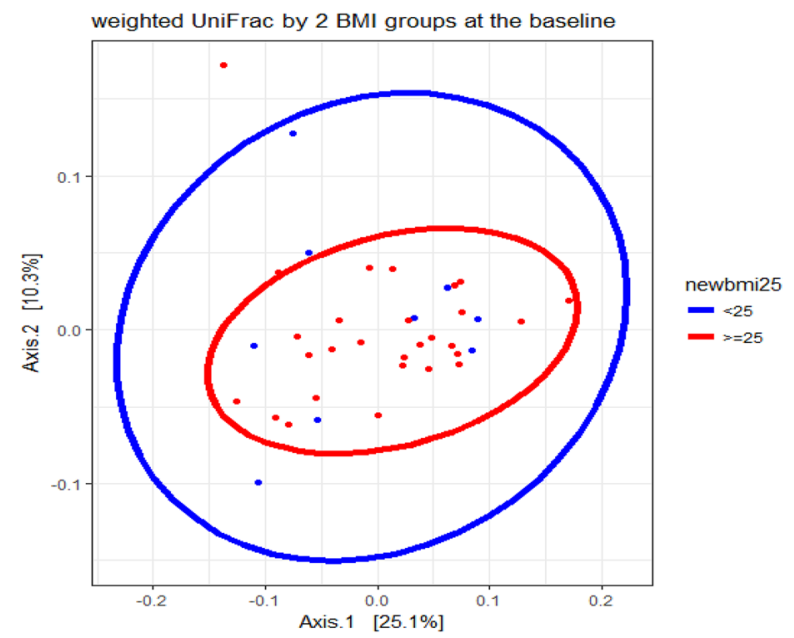

$20.9 \%$ while axis 2 explained $10.5 \%$ of the variance. B Weighted UniFrac-based principal component of the first two principal coordinates categorized by BMI; axis 1 explained $25.1 \%$ and axis 2 explained $10.3 \%$ of the variance 
Table 2 Median baseline alpha diversity measures ${ }^{\mathrm{a}}$ by select tumor characteristics and breast cancer risk factors

\begin{tabular}{|c|c|c|c|c|c|}
\hline & $N$ & Observed species & Chao1 & Shannon & PD tree \\
\hline \multicolumn{6}{|l|}{ Age } \\
\hline$<50$ & 20 & 37.50 & 69.05 & 3.07 & 12.65 \\
\hline $50+$ & 17 & 35.00 & 66.00 & 3.00 & 11.97 \\
\hline$p$ value & & 0.39 & 0.43 & 0.86 & 0.17 \\
\hline \multicolumn{6}{|l|}{ Stage } \\
\hline I/II & 22 & 34.00 & 56.06 & 2.98 & 11.79 \\
\hline III & 15 & 35.00 & 68.50 & 2.98 & 11.97 \\
\hline$p$ value $\mathrm{e}^{\mathrm{b}}$ & & 0.84 & 0.80 & 0.38 & 0.65 \\
\hline \multicolumn{6}{|l|}{ Grade } \\
\hline I/II & 14 & 31.00 & 54.00 & 2.97 & 11.46 \\
\hline III & 23 & 36.50 & 67.33 & 2.99 & 12.30 \\
\hline$p$ value $\mathrm{e}^{\mathrm{b}}$ & & 0.25 & 0.40 & 0.46 & 0.33 \\
\hline \multicolumn{6}{|l|}{ ER status } \\
\hline Positive & 28 & 33.50 & 60.86 & 2.97 & 11.72 \\
\hline Negative & 9 & 36.00 & 66.00 & 2.99 & 12.62 \\
\hline$p$ value $^{\mathrm{b}}$ & & 0.64 & 0.87 & 0.53 & 0.36 \\
\hline \multicolumn{6}{|l|}{ PR status } \\
\hline Positive & 23 & 33.50 & 57.49 & 2.97 & 11.72 \\
\hline Negative & 14 & 36.00 & 68.50 & 2.99 & 12.62 \\
\hline$p$ value $^{\mathrm{b}}$ & & 0.93 & 0.93 & 0.93 & 0.62 \\
\hline \multicolumn{6}{|l|}{ HER2 status } \\
\hline Positive & 12 & 26.00 & 53.00 & 2.71 & 10.88 \\
\hline Negative & 25 & 36.50 & 69.17 & 3.07 & 12.42 \\
\hline$p$ value $^{\mathrm{b}}$ & & 0.033 & 0.073 & 0.035 & 0.11 \\
\hline \multicolumn{6}{|l|}{ BMI $\left(\mathrm{kg} / \mathrm{m}^{2}\right)$} \\
\hline$<25$ & 9 & 38.00 & 71.58 & 3.12 & 12.49 \\
\hline$\geq 25$ & 28 & 33.00 & 58.13 & 2.92 & 11.97 \\
\hline$p$ value $^{\mathrm{b}}$ & & 0.091 & 0.24 & 0.11 & 0.33 \\
\hline \multicolumn{6}{|c|}{ Total body fat (TBF) } \\
\hline$\leq 46 \%$ & 25 & 36.52 & 72.41 & 3.03 & 12.11 \\
\hline$>46 \%$ & 12 & 31.17 & 49.99 & 2.91 & 11.05 \\
\hline$p$ value $^{\mathrm{b}}$ & & 0.059 & 0.011 & 0.35 & 0.26 \\
\hline \multicolumn{6}{|l|}{ BMI \&TBF } \\
\hline $\mathrm{I}(<25 \& \leq 46)$ & 9 & 38.56 & 74.67 & 3.16 & 12.34 \\
\hline $\mathrm{II}(\geq 25 \& \leq 46)$ & 16 & 35.38 & 71.13 & 2.96 & 11.97 \\
\hline III ( $\geq 25 \&>46)$ & 12 & 31.17 & 49.99 & 2.91 & 11.05 \\
\hline$p(2 \mathrm{df})$ & & 0.11 & 0.038 & 0.38 & 0.50 \\
\hline \multicolumn{6}{|l|}{ Age menarche } \\
\hline$\leq 11$ & 11 & 29.18 & 50.51 & 2.73 & 10.25 \\
\hline$\geq 12$ & 26 & 35.38 & 69.74 & 3.00 & 11.89 \\
\hline$p$ value $^{\mathrm{b}}$ & & 0.034 & 0.020 & 0.057 & 0.073 \\
\hline \multicolumn{6}{|l|}{ Livebirths } \\
\hline None & 8 & 35.5 & 51.8 & 3.04 & 11.72 \\
\hline $1+$ & 29 & 33.0 & 66.0 & 2.89 & 12.11 \\
\hline$p$ value $^{\mathrm{b}}$ & & 0.81 & 0.77 & 0.91 & 0.71 \\
\hline \multicolumn{6}{|l|}{ Physical activity ${ }^{\mathrm{c}}$} \\
\hline No & 13 & 34.00 & 48.75 & 3.11 & 12.53 \\
\hline Yes & 24 & 37.00 & 69.17 & 3.00 & 12.30 \\
\hline$p$ value $^{\mathrm{b}}$ & & 0.58 & 0.07 & 0.82 & 0.31 \\
\hline
\end{tabular}

${ }^{\mathrm{a}}$ Rarefaction of 100

${ }^{\mathrm{b}} p$ obtained by Wilcoxon rank sum test

${ }^{\mathrm{c}}$ No strenuous, vigorous or moderate activity per week

\section{Phyla abundance differences by tumor characteristics and breast cancer risk factors}

There were no significant phyla differences by ER and PR status, stage, grade, parity, BMI, and TBF\% (data not shown). However, median level of Firmicutes was lower among women with HER2 + than those with HER2- breast cancer ( 33.53 vs $51.75, p=0.005)$, and also lower among women with early $(\leq 11)$ than those with later $(\geq 12)$ age of menarche (35.61 vs 50.17, $p=0.048$ ) (Fig. 3). We explored differences in abundance by age at menarche and HER2 status combined (Fig. 4). Levels of Firmicutes were highest among those who had HER2 - and menarche age $\geq 12$ (56.24\%), intermediate among those who had HER2 - and menarche age $\leq 11(50.03 \%)$ or HER $2+$ and menarche age $\geq 12(30.4 \%)$, and lowest among those with HER2+ and menarche age $\leq 11(21.4 \%)\left(\mathrm{p}_{3 \mathrm{df}}=0.009\right)$. These results suggest an association of HER2 status with levels of Firmicutes among those with age at menarche at $\geq 12(p=0.027)$, and a borderline association of age at menarche with Firmicutes among women with HER2 - breast cancer $(p=0.105)$. The largest difference was between those who differed by both HER 2 status and age at menarche $(56.24 \%$ vs $21.4 \%$, $p=0.006$ ).

\section{Taxa abundance differences by ER, PR, and HER2 status}

Table 3 results showed MERs that differed significantly by ER, PR and HER2 status after adjusting for total counts, age, and race/ethnicity. MER $>1$ denotes higher taxa abundances in ER - than ER+, PR - than PR+, and HER2+ than HER2 - breast cancers whereas MER $<1$ shows lower taxa abundances in ER - than ER+, PR - than PR+, and HER2+ than HER 2- breast cancers. In total, 13 taxa differed between those with HER2+ vs HER2 - tumors $(p \leq 0.001)$, 3 taxa differed between ER+ and ER- tumors, and 2 taxa differed between PR+ and PR- tumors. The taxa that differed between HER2+ vs HER2 - tumors included specific Bacteroidetes (g_Alistipes), Firmicutes (g_Enterococcus, g_Acidaminococcus) showing higher abundances (MER $>1$ ) in HER2 + than HER2-. Other Bacteroidetes ( $f$ _Rikenellaceae), Euryarchaeto ( $g \_$Methanobrevibacter), Firmicutes (f_Christensenellaceae, g_Turicibacter, g_Clostridium, $g_{-}$ SMB53,g_Blautia, g_Coprococcus, g_Ruminococcus), and Proteobacteria (g_Desulfovibrio) showed lower abundances in HER2 + than HER2 - tumors. Abundance of three Firmicutes taxa (g_Enterococcus, g_Turicibacter, g_Veillonella) and one Proteobacteria taxa ( $g \_$Haemophilus) were lower in ER+ than ER-. Three Firmicutes taxa (g_Turicibacter, f_Clostridiaceae:g_Clostridium, $f_{-}$Erysipelotrichaceae:g Clostridium) were lower in PR+ than PR- breast cancers. The unadjusted relative abundances of select Firmicutes by 

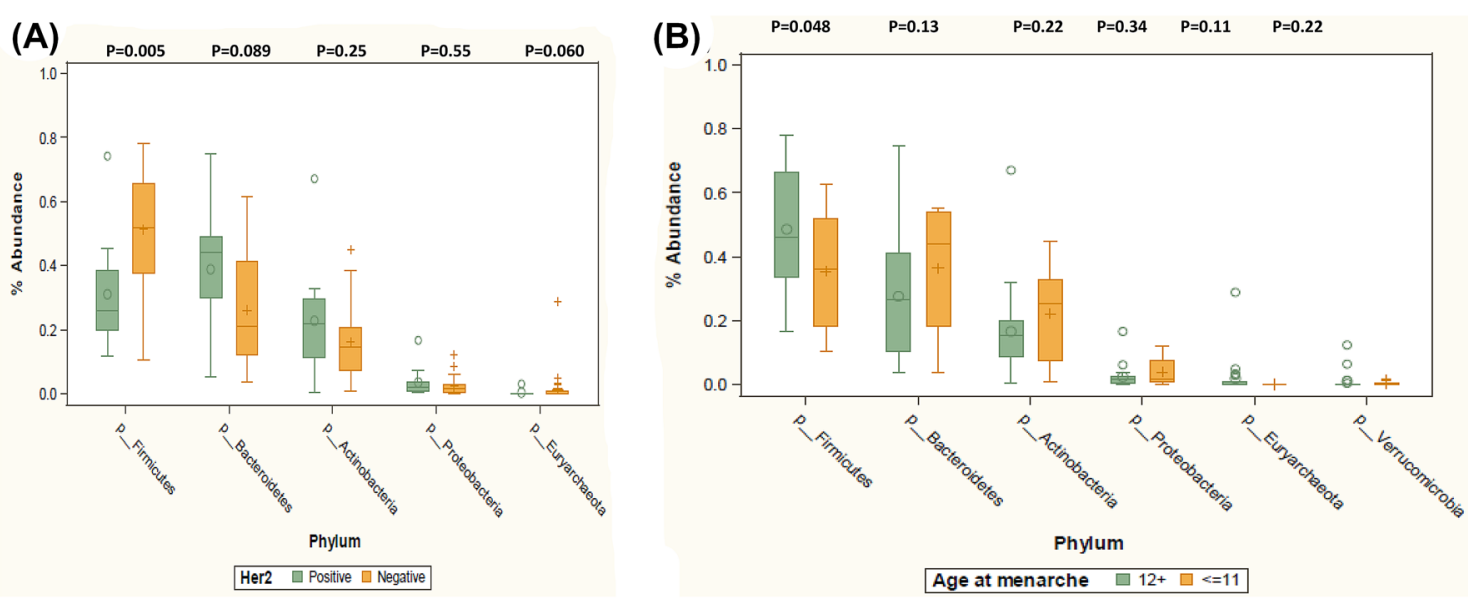

Fig. 3 Relative abundance levels of the most frequent phyla among A breast cancer patients with HER2+ tumors $(n=12)$ vs HER2tumors $(n=25)$, and $\mathbf{B}$ breast cancer patients with early age at menarche $(\leq 11)(n=11)$ vs later age at menarche $(\geq 12)(n=26)$ are

shown. Wilcoxon rank sum test was used to test for phylum-level differences by HER 2 status and by age at menarche. $p$ values are listed above each phylum
Fig. 4 Relative abundance levels (mean, median, minimum and maximum) of Firmicutes by four groups of breast cancer patients are shown: HER2breast cancer and later age at menarche $(\geq 12)(n=18)$, HER $2+$ breast cancer and late age at menarche $(n=8)$, HERbreast cancer and early age at menarche $(\leq 11)(n=7)$, and HER $2+$ breast cancer and early age at menarche $(n=4)$

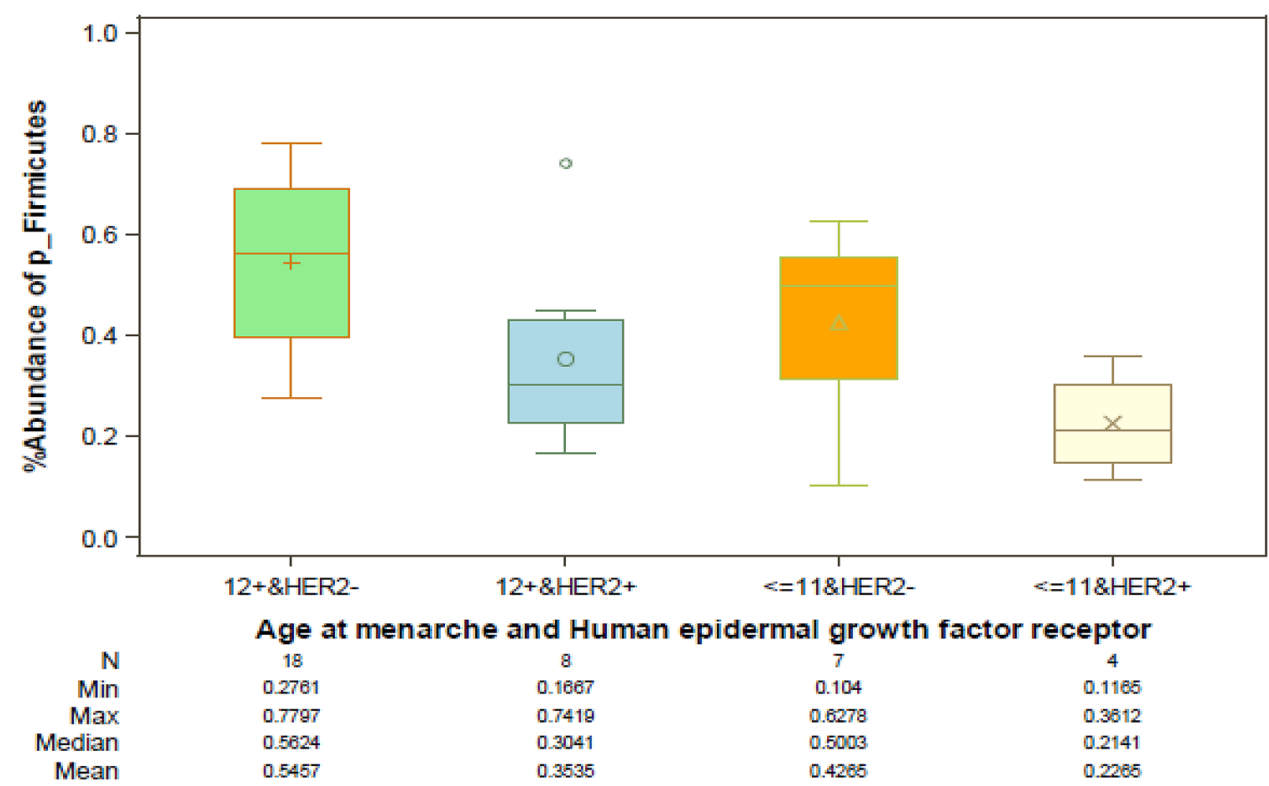

HER2 status are displayed in Fig. 5, in support of the results shown by MER in Table 3.

\section{Taxa abundance differences by stage and grade}

Two taxa of Firmicutes (g_Clostridium, g_Veillonella) were more abundant (MER > 1) among women with higher grade (III) or higher stage breast cancers compared to lower grade (I/II) or lower stage breast cancers. In addition, higher grade was associated with higher abundance of Actinobacteria ( $g_{-}$ Eggerthella) but lower abundance (MER $<1$ ) of other taxa of Actinobacteria (f_Coriobacteriaceae), and Firmucutes (f_Lachnospiraceae, g_Anaerostipes, f_Ruminococcaceae)
(Table 4). Higher stage breast cancer was also associated with higher abundance of Firmicutes (f_Clostridiaceae) and Proteobacteria (f_Enterobacteriaceae, g_Haemophilus) but lower abundance of Firmicutes (g_Acidaminococcus, g_Catenbacterium) (Table 4).

\section{Taxa abundance differences and breast cancer risk factors}

We also explored whether there are taxa differences by treating older age at diagnosis ( $\geq 50$ years), later age at menarche, parous, BMI $\left(<25 \mathrm{~kg} / \mathrm{m}^{2}\right)$, TBF $(\leq 46 \%)$, and physically active as the reference groups in the NBR 
Table 3 Mean ratio estimates (MER) $)^{\mathrm{a}}$ obtained by zero-inflated negative binomial model of taxa abundances by estrogen receptor (ER), progesterone receptor (PR), and human epidermal growth factor receptor 2 (HER2) status with adjustment for total counts, age and race/ethnicity (model 2, MER)

\begin{tabular}{|c|c|c|c|c|c|c|c|c|}
\hline & & & \multicolumn{2}{|c|}{ ER- vs ER+ } & \multicolumn{2}{|c|}{ PR- vs PR+ } & \multicolumn{2}{|c|}{$\begin{array}{l}\text { HER2+ vs } \\
\text { HER2- }\end{array}$} \\
\hline & & & MER & $\begin{array}{l}p \\
\text { value }\end{array}$ & MER & $\begin{array}{l}p \\
\text { value }\end{array}$ & MER & $\begin{array}{l}p \\
\text { value }\end{array}$ \\
\hline \multicolumn{9}{|l|}{ p__Bacteroidetes } \\
\hline & f__Rikenellaceae & & & & & & .039 & .0060 \\
\hline & f__Rikenellaceae & g__Alistipes & & & & & 4.953 & .0075 \\
\hline \multicolumn{9}{|l|}{ p__Euryarchaeota } \\
\hline & f_Methanobacteriaceae & g_Methanobrevibacter & & & & & .001 & .0039 \\
\hline \multicolumn{9}{|l|}{$\mathrm{p} \_$Firmicutes } \\
\hline & f_Enterococcaceae & g__Enterococcus & .045 & .0037 & & & 59.538 & .0012 \\
\hline & f__Turicibacteraceae & g__Turicibacter & .034 & .0092 & .114 & .0031 & .157 & .0050 \\
\hline & f__Chistenseneitaceae & $g_{-}$ & & & & & .085 & .0002 \\
\hline & f__Clostridiaceae & g__Clostridium & & & .184 & .0015 & .165 & .0023 \\
\hline & f__Clostridiaceae & g__SMB53 & & & & & .214 & .0046 \\
\hline & f__Lachnospiraceae & g__Blautia & & & & & .409 & .0085 \\
\hline & f__Lachnospiraceae & g__Coprococcus & & & & & .405 & .0077 \\
\hline & f__Lachnospiraceae & $\mathrm{g}$ _[Ruminococcus] & & & & & .287 & .0002 \\
\hline & $\mathrm{f} \_$Veillonellaceae & g_Acidaminococcus & & & & & 244.94 & .0003 \\
\hline & f__Veillonellaceae & g__Veillonella & .074 & .0003 & & & & \\
\hline & f__Erysipelotrichaceae & g__Clostridium & & & .058 & .0051 & & \\
\hline \multicolumn{9}{|l|}{$\mathrm{p} \_$Proteobacteria } \\
\hline & f__Desulfovibrionaceae & g__Desulfovibrio & & & & & .059 & .0005 \\
\hline & f__Pasteurellaceae & g_Haemophilus & .014 & $<.0001$ & & & & \\
\hline
\end{tabular}

${ }^{a}$ MER > 1 means higher taxa in ER- than ER+, PR - than PR+, and HER2+ than HER2- group; ER+, PR+, and HER2- was the respective reference group

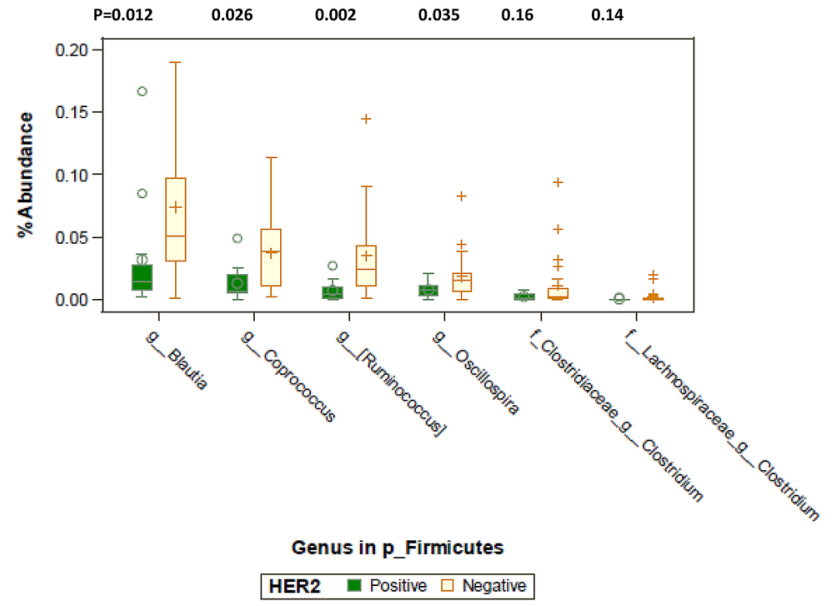

Fig. 5 Relative abundance levels of select genera of Firmicutes by HER2 status are shown. Wilcoxon rank sum test was used to test for genus-level differences by HER 2 status. $p$ values are listed above each genus

model analysis (Tables 5 and 6). Younger women at diagnosis ( $<50$ years) (higher risk) compared to older age at diagnosis displayed higher abundance (MER $>1)$ in five taxa including Actinobacteria ( $g$ Eggerthella) and Firmicutes (f_Clostridiaceae, g_SMB53,g_Clostridium, g_Lactococcus). Women who reported menarche age $\leq 11$ (higher risk) compared to $\geq 12$ menarche age showed significant differences in nine taxa, including lower abundance (MER $<1)$ of Actinobacteria ( $f$ Coriobacteriaceae), Euryarchaeota (g_Methanobrevibacter) and Firmicutes (g_Turicibacter, $g \_$Anaerostipes, g_Lachnobacterium, $f \_$Ruminococcaceae, $g_{-}$Ruminococcus) but higher abundance (MER > 1) of Firmicutes (f_Lachnospiracaceae:g_Clostridium) and Proteobacteria (g_Escherichla). Nulliparous compared with parous women displayed lower abundance $(\mathrm{MER}<1)$ of two genera of Firmicutes ( $g \_$Lactococcus, g_Catenibacterium) but higher abundance (MER > 1) of Actinobacteria (g_Actinomyces) and Proteobacteria (g_Bilophila).

Differences in select taxa emerged in comparisons by BMI $\left(<25 \mathrm{vs} \geq 25 \mathrm{~kg} / \mathrm{m}^{2}\right)$ and $\mathrm{TBF}(<46 \% \mathrm{vs} \geq 46 \%)$; BMI and TBF were highly correlated $\left(\mathrm{R}^{2}=0.61, p<0.0001\right)$ (Table 6 ). Women with BMI $\geq 25 \mathrm{~kg} / \mathrm{m}^{2}$ compared to those with lower BMI displayed higher abundance (MER $>1$ ) of Firmicutes (f_Clostridiaceae) and Verrucomicrobia (g_Akkermansia) 
Table 4 Mean estimate ratios $(\mathrm{MER})^{\mathrm{a}}$ obtained by zeroinflated negative binomial model of taxa abundances by grade and stage of breast cancer with adjustment for total counts, age and race/ethnicity (model 2, MER)

\begin{tabular}{|c|c|c|c|c|c|c|}
\hline & & & \multicolumn{2}{|c|}{$\begin{array}{l}\text { Grade high (III) } \\
\text { vs low (I/II) }\end{array}$} & \multicolumn{2}{|c|}{$\begin{array}{l}\text { Stage high (III) } \\
\text { vs low (I/II) }\end{array}$} \\
\hline & & & MER & $p$ value & MER & $p$ value \\
\hline \multicolumn{7}{|l|}{$\mathrm{p} \_$Actinobacteria } \\
\hline & f__Coriobacteriaceae & $\mathrm{g}$ & .238 & 0.0028 & & \\
\hline & f__Coriobacteriaceae & g__Eggerthella & 9.365 & 0.0004 & & \\
\hline \multicolumn{7}{|l|}{$\mathrm{p} \_$_Firmicutes } \\
\hline & f__Clostridiaceae & $\mathrm{g}$ & & & 3.290 & .0011 \\
\hline & f__Clostridiaceae & g_Clostridium & 6.144 & 0.0088 & 5.986 & .0005 \\
\hline & f__Lachnospiraceae & & .343 & 0.0003 & & \\
\hline & f__Lachnospiraceae & g__Anaerostipes & .116 & $<0.0001$ & & \\
\hline & f__Ruminococcaceae & & .488 & 0.0066 & & \\
\hline & $\mathrm{f} \_$Veillonellaceae & g_Acidaminococcus & & & .0098 & .0003 \\
\hline & f__Veillonellaceae & g__Veillonella & 9.794 & 0.0025 & 15.12 & $<.0001$ \\
\hline & f__Erysipelotrichaceae & g_Catenibacterium & & & .151 & .0002 \\
\hline \multicolumn{7}{|l|}{$\mathrm{p} \_$_Proteobacteria } \\
\hline & f__Enterobacteriaceae & & & & 6.389 & .0024 \\
\hline & f__Pasteurellaceae & g_Haemophilus & & & 71.633 & $<.0001$ \\
\hline
\end{tabular}

${ }^{a}$ MER > 1 means higher taxa in high grade (III) than low grade (I and II) and in high stage (III) than low stage (I and II); low grade and low stage was the respective reference group but lower abundance $($ MER $<1)$ of Firmicutes ( $g_{-}$Lactobacillus, g_Streptococcus). When we examined difference in taxa by TBF, women with higher TBF $(\geq 46 \%)$ compared to those with lower $\operatorname{TBF}(<46 \%)$ also showed higher abundance (MER $>1$ ) of Firmicutes ( $f$ _Clostridiaceae, g_Clostridium, g_Lachnospira) but lower abundance $(\mathrm{MER}<1)$ of Actinobacteria (f_Coriobacteriaceae) and Firmiciutes ( $g$ _Catenbacterium). There are some taxa differences between those who were physically active compared to those who were inactive; including lower abundance of some Firmicutes (f_Clostridiaceae; g_Lachnobacterium, g_Lactobacillus) but higher abundance of other Firmicutes ( $f_{-}$Veillonella).

\section{Discussion}

We investigated the gut microbiome profile in relation to ER/PR and HER2 status, tumor grade and stage, and select breast cancer risk factors in 37 women diagnosed with incident breast cancer; most of whom (73\%) were Hispanics, and were overweight or obese (75\%). Women with HER2+ compared with HER2- breast cancers displayed a less diverse microbiome and a distinct bacterial composition profile, including in abundance of Firmicutes (see below). Breast cancer patients with high $(\geq 46 \%) \mathrm{TBF}$ and earlier age at menarche $(\leq 11)$ also had a less diverse gut microbiome. Abundance of Firmicutes was significantly lower among women with HER2+ breast cancer and early menarche than those with HER2 - breast cancer and later menarche. Before we interpret these new results, we discuss our results on body size comparisons and tumor grade and stage in relation to published findings.

Alpha diversity measures have been used as a hallmark of health habits including adherence to Mediterranean diets [23-25] and body composition [26]. Lower gut alpha diversity has been associated with human obesity in a metaanalysis, showing significant relationships between obesity and microbial richness, evenness, and diversity [26]. Chao 1 index and OTU were $31 \%(p=0.011)$ and $14 \%(p=0.059)$ lower among women with $>46 \% \mathrm{TBF}$ compared to those with $\leq 46 \%$ TBF; similar but weaker patterns were observed by BMI (Table 2). Associations between various bacterial groups and BMI have been reported but a consistent taxonomic signature of obesity has not been identified [27, 28]. Women in this study with higher BMI or higher TBF displayed higher abundance of Firmicutes (f_Clostridiaceae). Additionally, those with higher BMI displayed higher abundance of $g_{-}$Akkermansia; enrichment of this taxa has been related with body composition in other studies [29-31]. Several sub-taxa within Firmicutes (g_Streptococcus) associated with lower BMI [28, 31, 32] also appeared to differ by BMI in this study. However, small numbers of those with $\mathrm{BMI}<25 \mathrm{~kg} / \mathrm{m}^{2}(n=9)$ may have limited our ability to identify other taxa that have been associated with lean/normal BMI (e.g., f_Christensenellaceae; g_Oscillospira) [23, $33,34]$. Interestingly, breast cancer patients without regular physical activity also showed lower Chao 1 index $(p=0.07)$ and tended to have lower abundance of several taxa of Firmicutes (f_Clostridiaceae) in support of growing evidence that exercise favorably influences the function and composition 
Table 5 Mean estimate ratios (MER) ${ }^{\mathrm{a}}$ obtained by zero-inflated negative binomial model of taxa abundances by age group ${ }^{\mathrm{b}}$, menarche age and parity $^{\mathrm{c}}$

\begin{tabular}{|c|c|c|c|c|c|c|c|c|}
\hline & & & \multicolumn{2}{|c|}{$\operatorname{Age}(\leq 50 \text { vs } 50+)^{\mathrm{b}}$} & \multicolumn{2}{|c|}{$\begin{array}{l}\text { Menarche Age } \\
\leq 11 \text { vs } \geq 12\end{array}$} & \multicolumn{2}{|c|}{$\begin{array}{l}\text { Nulliparous vs } \\
\text { Parous }^{c}\end{array}$} \\
\hline & & & MER & $p$ value & MER & $p$ value & MER & $p$ value \\
\hline \multicolumn{9}{|l|}{ p__Actinobacteria } \\
\hline & f__Actinomycetaceae & g_Actinomyces & & & & & 4.006 & .0068 \\
\hline & f_Coriobacteriaceae & g__Eggerthella & 6.0133 & 0.002 & & & & \\
\hline & f_Coriobacteriaceae & $\mathrm{g}$ & & & .2447 & .0062 & & \\
\hline \multicolumn{9}{|l|}{$\mathrm{p} \_$_Bacteroidetes } \\
\hline & f__Methanobacteriaceae & g__Methanobrevibacter & & & .0081 & .001 & & \\
\hline \multicolumn{9}{|l|}{$\mathrm{p} \_$_Firmicutes } \\
\hline & f__Clostridiaceae & & 12.5643 & $<.0001$ & & & & \\
\hline & f__Clostridiaceae & g__SMB53 & 7.2232 & 0.0068 & & & & \\
\hline & f__Erysipelotrichaceae & g_Catenibacterium & & & & & .0104 & .0001 \\
\hline & f__Erysipelotrichaceae & g_Clostridium & 19.9947 & $<.0001$ & & & & \\
\hline & f__Lachnospiraceae & g__Anaerostipes & & & .0403 & .0029 & & \\
\hline & f__Lachnospiraceae & g_Clostridium & & & 8.280 & .0086 & & \\
\hline & f__Lachnospiraceae & g_Lachnobacterium & & & .0143 & .0011 & & \\
\hline & f__Streptococcaceae & g_L_Lactococcus & 32.8322 & $<.0001$ & & & .0419 & .0055 \\
\hline & f__Turicibacteraceae & g_turicibacter & & & .0874 & .0028 & & \\
\hline & f__Ruminococcaceae & & & & .4229 & .0016 & & \\
\hline & f__Ruminococcaceae & g_Ruminococcus & & & .2068 & .0004 & & \\
\hline \multicolumn{9}{|l|}{ P_Proteobacteria } \\
\hline & f__Enterobacteriaceae & & 10.4271 & 0.0005 & & & & \\
\hline & f__Desulfovibrionaceae & g__Bilophila & & & & & 3.0562 & .0064 \\
\hline & $\mathrm{f} \_$Enterobacteriaceae & g_Escherichla & & & 31.523 & $<0.0001$ & & \\
\hline
\end{tabular}

${ }^{\mathrm{a}}$ MER $>$ means higher taxa in women aged $<50$, early menarche age $(\leq 11)$, nulliparous, high BMI $(\geq 25)$, high $\mathrm{TBF}(>46 \%)$ than age $50+$, later menarche $(\geq 12)$, parous, low BMI, and low TBF, respectively

${ }^{\mathrm{b}}$ Adjustment for total counts and race/ethnicity

${ }^{\mathrm{c}}$ Adjustment for total counts, age and race/ethnicity in analysis on age at menarche and parity (model 2, MER)

of human gut microbiota] [35] However, limited sample size precluded our ability to examine the combined effects of physical activity and finer categories of BMI on microbiome diversity and composition. Results from a large study showed that microbiome differences by BMI may be missed if categories of BMI comparisons are crude. In this previous study, microbiome composition did not differ between normal weight $\left(<25 \mathrm{~kg} / \mathrm{m}^{2}\right)$ and overweight $\left(25-30 \mathrm{~kg} / \mathrm{m}^{2}\right)$ persons, but there were significant differences in microbiome between normal weight and those who had class I obesity $(>30-\leq 35)$ or class II obesity $>35 \mathrm{~kg} / \mathrm{m}^{2}$ [28].

Our findings on taxa differences by breast cancer grade and stage add to results from one previous study of mostly low grade (77\% were grade I/II) and low stage (59\% stage 0/I) breast cancers [7]. A higher abundance of $g_{-}$Clostridium was found among those with higher tumor grade or stage in this study, similar to the finding of abundance of Clostridium coccoides cluster in the previous study [7]. Moreover, women with higher grade or higher stage breast cancers also displayed higher abundance of $f_{-}$Veillonella but lower abundance of $f_{-}$Erysipelotrichaeceae which has been related with inflammation-related conditions [36]. The significance of our finding of high abundance of taxa in $p_{-}$Proteobacteria (g_Haemophilus, $f \_$Enterobacteriaceae) among those with higher tumor stage is not clear but it is intriguing that g_Haemophilus appeared to be over-represented among individuals with impaired glucose regulation [36].

Reasons for the lower alpha diversity among women with HER2+ compared to those with HER2- breast cancer are not known. Menarche age, parity, BMI, and TBF did not differ by HER2 status. It is intriguing that women with HER2+ compared to those with HER2 - breast cancer displayed lower abundance of select genera of Firmicutes (e.g., g_Clostridium, g_Blautia, g_Coprococcus, g_Ruminococcus, g_SMB53) and higher abundance of select genera of $p_{-}$Bacteroidetes; thus a deficit of taxa that have often been linked with healthy body composition, body leanness and healthy metabolic profile [37, 38]. 
Table 6 Mean estimate ratios (MER) ${ }^{\mathrm{a}}$ obtained by zero-inflated negative binomial model of taxa abundances by BMI, total body fat, and physical activity with adjustment for total counts, age and race/ethnicity (model 2, MER)

\begin{tabular}{|c|c|c|c|c|c|c|c|c|}
\hline & & & \multicolumn{2}{|c|}{$\begin{array}{l}\text { BMI }\left(\mathrm{kg} / \mathrm{m}^{2}\right) \\
\geq 25 \text { vs }<25\end{array}$} & \multicolumn{2}{|c|}{$\begin{array}{l}\text { Total body fat } \\
\text { (TBF) } \\
>46 \% \text { vs } \leq 46 \%\end{array}$} & \multicolumn{2}{|c|}{$\begin{array}{l}\text { Physical activity } \\
\text { (none vs yes) }\end{array}$} \\
\hline & & & MER & $p$ value & MER & $p$ value & MER & $p$ value \\
\hline \multicolumn{9}{|l|}{$\mathrm{p} \_$Actinobacteria } \\
\hline & f__Coriobacteriaceae & $\mathrm{g}$ & & & .0661 & $<.0001$ & 0.1418 & 0.0004 \\
\hline \multicolumn{9}{|l|}{$\mathrm{p} \_$_Firmicutes } \\
\hline & f__Lactobacillaceae & g__Lactobacillus & .053 & .0083 & & & & \\
\hline & f__Streptococcaceae & g_Streptococcus & .134 & .0012 & & & & \\
\hline & f__Clostridiaceae & & 1.985 & .0074 & 7.909 & $<.0001$ & 0.1023 & $<.0001$ \\
\hline & f__Clostridiaceae & g__Clostridium & & & 6.901 & .0033 & & \\
\hline & f__Lachnospiraceae & g_Lachnobacterium & & & & & 0.0275 & 0.0004 \\
\hline & f__Lactobacillaceae & g__Lactobacillus & & & & & 0.0388 & 0.0015 \\
\hline & f__Lachnospiraceae & g_Lachnospira & & & 3.127 & .0085 & & \\
\hline & f__Veillonellaceae & g__Veillonella & & & & & 12.3926 & 0.0007 \\
\hline & f__Erysipelotrichaceae & g_Catenibacterium & & & .0809 & .002 & & \\
\hline \multicolumn{9}{|l|}{$\mathrm{p} \_$Verrucomicrobia } \\
\hline & f__Verrucomicrobiaceae & g__Akkermansia & 181.63 & $<0.0001$ & & & & \\
\hline
\end{tabular}

${ }^{\mathrm{a}}$ MER $>$ means higher taxa in high BMI $(\geq 25)$, high $\mathrm{TBF}(>46 \%)$, and no regular physical activity than low BMI, and low TBF and yes regular physical activity

Lower weight gain has been associated with taxa of the Ruminococcaceae family in studies of twins [27].

Another novel finding is that earlier menarche age was associated with lower alpha diversity; these findings were statistically significant for OTU and Chao1 index. Age at menarche is likely a marker of earlier life diet and nutrition [39]. Earlier age at menarche has been found to have a lasting effect [40], conferring higher circulating estradiol levels for those who started to menstruate at ages 11 or younger than at age 14 or older $(p=0.033)$ [41]. High gut microbial diversity has been associated with a profile of estrogen metabolites associated with reduced breast cancer risk [42]. Levels of urinary estrogen metabolites have been correlated with relative abundances of specific Clostridia taxa [42, 43]. There are likely bidirectional influences between sex steroids and the gut microbiome. Various bacterial genes have been found to affect $\beta$-glucuronidase enzymatic activity, influencing deconjugation and reabsorption of estrogens. Levels of circulating estrogen, in turn, may influence the abundance of certain bacteria species [42-47].

Strengths of this pilot study include our collection of detailed information on relevant breast cancer risk factors and tumor characteristics and considering them in this analysis using two complementary methods, by Wilcoxon rank sum test and a zero-inflated NBR model with adjustment for select covariates. This study included mostly Hispanics in the catchment area of USC. However, we are limited by our cross-sectional analyses and modest sample size so that we used only two categories in our comparisons of taxa differences by age at menarche, parity, physical activity, BMI and TBF\%. Breastfeeding, a parity-related factor, that has emerged as an important modifiable lifestyle factor for breast cancer, was not asked in our study. Research regarding the association of specific microbiome taxa to disease or other conditions inherently involves studying the relationships of numerous taxa with multiple conditions, thus greatly increasing the possibility of type 1 errors. On the other hand, small sample sizes preclude the recognition of any but the strongest associations when very small alphalevels are used for statistical significance. Even with our conservative $\alpha$-level of 0.001 we found far more statistically significant results than would be expected by chance alone, particularly with respect to HER2, grade, and age at menarche. Although some of these findings may be chance findings, while other important associations may have been missed due to the small alpha used, we feel that we have struck a reasonable balance, and that these findings are informative and warrant further consideration.

\section{Conclusions}

In summary, this pilot cross-sectional study of mostly Hispanic women found that HER2 status and age at menarche had significant associations with gut microbiome alpha diversity measures and specific microbial composition. These findings warrant confirmation in studies with larger 
sample sizes of diverse racial/ethnic groups and with repeated sample collections to determine how microbiome are associated with breast cancer subtypes and specific risk factors.

Acknowledgements We gratefully acknowledge all the women who participated in this study, Wendy Cheng for coordinating this study, Diano Chingos (patient advocate) for supportive comments and advice and Dr. Jacques Ravel and Dr. Mike Humphrys at the University of Maryland for overseeing the 16S rRNA gene sequencing for this study.

Author contributions AHW conceived the study and obtained funding with advice from CV, DS, AAG and WC. DS and AAG supervised the recruitment of patients. CT and CV performed the statistical analyses. $\mathrm{AHW}, \mathrm{CT}, \mathrm{CV}$, and $\mathrm{YY}$ interpreted the data. AHW and CV were the primary contributors to the manuscript. All authors approved the final manuscript.

Funding This study was supported by the California Breast Research Program Grants (20IB-0105) and the USC Norris Comprehensive Cancer Center Core Support Grant (P30 CA14089) (Wu). The funders of the study had no role in study design; in the collection, analysis or interpretation of the data, or the writing this manuscript. AHW had full access to all study data and final responsibility to submit for publication.

\section{Compliance with ethical standards}

Conflict of interest The authors declare that they have no conflict of interest.

Research involving human participants and/or animals All procedures performed in studies involving human participants were in accordance with the ethical standards of the institutional and/or national research committee and with the 1964 Helsinki declaration and its later amendments or comparable ethical standards. The study protocol was approved by the USC Institutional Review Board.

Informed consent Informed consent was obtained from all individual participants included in this study.

Open Access This article is licensed under a Creative Commons Attribution 4.0 International License, which permits use, sharing, adaptation, distribution and reproduction in any medium or format, as long as you give appropriate credit to the original author(s) and the source, provide a link to the Creative Commons licence, and indicate if changes were made. The images or other third party material in this article are included in the article's Creative Commons licence, unless indicated otherwise in a credit line to the material. If material is not included in the article's Creative Commons licence and your intended use is not permitted by statutory regulation or exceeds the permitted use, you will need to obtain permission directly from the copyright holder. To view a copy of this licence, visit http://creativecommons.org/licenses/by/4.0/.

\section{References}

1. Ley RE, Peterson DA, Gordon JI (2006) Ecological and evolutionary forces shaping microbial diversity in the human intestine. Cell 124(4):837-848

2. Ley RE (2010) Obesity and the human microbiome. Curr Opin Gastroenterol 26(1):5-11
3. Fernandez MF, Reina-Perez I, Astorga JM, Rodriguez-Carrillo A, Plaza-Diaz J, Fontana L (2018) Breast cancer and its relationship with the microbiota. Int J Environ Res Public Health 15(8): 1747

4. Parida S, Sharma D (2019) The power of small changes: comprehensive analyses of microbial dysbiosis in breast cancer. Biochim Biophys Acta Rev Cancer 1871(2):392-405

5. Goedert JJ, Jones G, Hua X, Xu X, Yu G, Flores R, Falk RT, Gail MH, Shi J, Ravel J, Feigelson HS (2015) Investigation of the association between the fecal microbiota and breast cancer in postmenopausal women: a population-based case-control pilot study. J Natl Cancer Inst. https://doi.org/10.1093/jnci/djv147

6. Yang J, Tan Q, Fu Q, Zhou Y, Hu Y, Tang S, Zhou Y, Zhang J, Qiu J, Lv Q (2016) Gastrointestinal microbiome and breast cancer: correlations, mechanisms and potential clinical implications. Breast Cancer 24:220-228

7. Luu TH, Michel C, Bard JM, Dravet F, Nazih H, BobinDubigeon C (2017) Intestinal proportion of blautia sp. is associated with clinical stage and histoprognostic grade in patients with early-stage breast cancer. Nutr Cancer 69(2):267-275

8. Ma H, Bernstein L, Pike MC, Ursin G (2006) Reproductive factors and breast cancer risk according to joint estrogen and progesterone receptor status: a meta-analysis of epidemiological studies. Breast Cancer Res 8(4):R43

9. Barnard ME, Boeke CE, Tamimi RM (2015) Established breast cancer risk factors and risk of intrinsic tumor subtypes. Biochim Biophys Acta 1856(1):73-85

10. Gaudet MM, Gierach GL, Carter BD, Luo J, Milne RL, Weiderpass E, Giles GG, Tamimi RM, Eliassen AH, Rosner B, Wolk A, Adami HO, Margolis KL, Gapstur SM, Garcia-Closas M, Brinton LA (2018) Pooled Analysis of nine cohorts reveals breast cancer risk factors by tumor molecular subtype. Cancer Res 78(20):6011-6021

11. Ma H, Ursin G, Xu X, Lee E, Togawa K, Duan L, Lu Y, Malone KE, Marchbanks PA, McDonald JA, Simon MS, Folger SG, Sullivan-Halley J, Deapen DM, Press MF, Bernstein L (2017) Reproductive factors and the risk of triple-negative breast cancer in white women and African-American women: a pooled analysis. Breast Cancer Res 19(1):6

12. Urbaniak C, Cummins J, Brackstone M, Macklaim JM, Gloor GB, Baban CK, Scott L, O'Hanlon DM, Burton JP, Francis KP, Tangney M, Reid G (2014) Microbiota of human breast tissue. Appl Environ Microbiol 80(10):3007-3014

13. Xuan C, Shamonki JM, Chung A, Dinome ML, Chung M, Sieling PA, Lee DJ (2014) Microbial dysbiosis is associated with human breast cancer. PLoS ONE 9(1):e83744

14. Chan AA, Bashir M, Rivas MN, Duvall K, Sieling PA, Pieber TR, Vaishampayan PA, Love SM, Lee DJ (2016) Characterization of the microbiome of nipple aspirate fluid of breast cancer survivors. Sci Rep 6:28061

15. Hieken TJ, Chen J, Hoskin TL, Walther-Antonio M, Johnson S, Ramaker S, Xiao J, Radisky DC, Knutson KL, Kalari KR, Yao JZ, Baddour LM, Chia N, Degnim AC (2016) The microbiome of aseptically collected human breast tissue in benign and malignant disease. Sci Rep 6:30751

16. Urbaniak C, Gloor GB, Brackstone M, Scott L, Tangney M, Reid G (2016) The microbiota of breast tissue and its association with breast cancer. Appl Environ Microbiol 82(16):5039-5048

17. Chiba A, Bawaneh A, Velazquez C, Clear KY, Wilson AS, Howard-McNatt M, Levine EA, Levi-Polyachenko N, Yates-Alston SA, Diggle SP, Soto-Pantoja DR, Cook KL (2019) Neoadjuvant chemotherapy shifts breast tumor microbiota populations to regulate drug responsiveness and the development of metastasis. Mol Cancer Res 18:130-139

18. Banerjee S, Tian T, Wei Z, Shih N, Feldman MD, Peck KN, DeMichele AM, Alwine JC, Robertson ES (2018) Distinct 
microbial signatures associated with different breast cancer types. Front Microbiol 9:951

19. Flores R, Shi J, Gail MH, Gajer P, Ravel J, Goedert JJ (2012) Assessment of the human faecal microbiota: II. Reproducibility and associations of $16 \mathrm{~S}$ rRNA pyrosequences. Eur J Clin Invest 42(8):855-863

20. Lozupone C, Knight R (2005) UniFrac: a new phylogenetic method for comparing microbial communities. Appl Environ Microbiol 71(12):8228-8235

21. Fang R, Wagner BD, Harris JK, Fillon SA (2016) Zero-inflated negative binomial mixed model: an application to two microbial organisms important in oesophagitis. Epidemiol Infect 144(11):2447-2455

22. Bender R, Lange S (2001) Adjusting for multiple testing-when and how? J Clin Epidemiol 54(4):343-349

23. Garcia-Mantrana I, Selma-Royo M, Alcantara C, Collado MC (2018) Shifts on gut microbiota associated to Mediterranean diet adherence and specific dietary intakes on general adult population. Front Microbiol 9:890

24. Lin D, Peters BA, Friedlander C, Freiman HJ, Goedert JJ, Sinha R, Miller G, Bernstein MA, Hayes RB, Ahn J (2018) Association of dietary fibre intake and gut microbiota in adults. Br J Nutr 120(9):1014-1022

25. Shively CA, Register TC, Appt SE, Clarkson TB, Uberseder B, Clear KYJ, Wilson AS, Chiba A, Tooze JA, Cook KL (2018) Consumption of Mediterranean versus western diet leads to distinct mammary gland microbiome populations. Cell Rep 25(1):47-56

26. Sze MA, Schloss PD (2016) Looking for a signal in the noise: revisiting obesity and the microbiome. MBio 7(4):e01018

27. Menni C, Jackson MA, Pallister T, Steves CJ, Spector TD, Valdes AM (2017) Gut microbiome diversity and high-fibre intake are related to lower long-term weight gain. Int J Obes (London) 41(7):1099-1105

28. Peters BA, Shapiro JA, Church TR, Miller G, Trinh-Shevrin C, Yuen E, Friedlander C, Hayes RB, Ahn J (2018) A taxonomic signature of obesity in a large study of American adults. Sci Rep 8(1):9749

29. Javurek AB, Spollen WG, Johnson SA, Bivens NJ, Bromert KH, Givan SA, Rosenfeld CS (2016) Effects of exposure to bisphenol A and ethinyl estradiol on the gut microbiota of parents and their offspring in a rodent model. Gut Microbes 7(6):471-485

30. Choi S, Hwang YJ, Shin MJ, Yi H (2017) Difference in the gut microbiome between ovariectomy-induced obesity and dietinduced obesity. J Microbiol Biotechnol 27(12):2228-2236

31. Fruge AD, Van der Pol W, Rogers LQ, Morrow CD, Tsuruta Y, Demark-Wahnefried W (2018) Fecal Akkermansia muciniphila is associated with body composition and microbiota diversity in overweight and obese women with breast cancer participating in a presurgical weight loss trial. J Acad Nutr Diet 120:650-659

32. Clarke SF, Murphy EF, Nilaweera K, Ross PR, Shanahan F, O'Toole PW, Cotter PD (2012) The gut microbiota and its relationship to diet and obesity: new insights. Gut Microbes 3(3): 186-202

33. Goodrich JK, Waters JL, Poole AC, Sutter JL, Koren O, Blekhman R, Beaumont M, Van Treuren W, Knight R, Bell JT, Spector TD, Clark AG, Ley RE (2014) Human genetics shape the gut microbiome. Cell 159(4):789-799

34. Waters JL, Ley RE (2019) The human gut bacteria Christensenellaceae are widespread, heritable, and associated with health. BMC Biol 17(1):83. https://doi.org/10.1186/s12915-019-0699-4
35. Cronin O, Molloy MG, Shanahan F (2016) Exercise, fitness, and the gut. Curr Opin Gastroenterol 32(2):67-73

36. Kaakoush NO (2015) Insights into the role of Erysipelotrichaceae in the human host. Front Cell Infect Microbiol 5:84

37. Konikoff T, Gophna U (2016) Oscillospira: a central, enigmatic component of the human gut microbiota. Trends Microbiol 24(7):523-524

38. Guo Y, Huang ZP, Liu CQ, Qi L, Sheng Y, Zou DJ (2018) Modulation of the gut microbiome: a systematic review of the effect of bariatric surgery. Eur J Endocrinol 178(1):43-56

39. Koprowski C, Ross RK, Mack WJ, Henderson BE, Bernstein L (1999) Diet, body size and menarche in a multiethnic cohort. $\mathrm{Br}$ J Cancer 79(11-12):1907-1911

40. Apter D, Reinila M, Vihko R (1989) Some endocrine characteristics of early menarche, a risk factor for breast cancer, are preserved into adulthood. Int J Cancer 44(5):783-787

41. Key TJ, Appleby PN, Reeves GK, Roddam AW, Helzlsouer KJ, Alberg AJ, Rollison DE, Dorgan JF, Brinton LA, Overvad K, Kaaks R, Trichopoulou A, Clavel-Chapelon F, Panico S, Duell EJ, Peeters PH, Rinaldi S, Fentiman IS, Dowsett M, Manjer J, Lenner P, Hallmans G, Baglietto L, English DR, Giles GG, Hopper JL, Severi G, Morris HA, Hankinson SE, Tworoger SS, Koenig K, Zeleniuch-Jacquotte A, Arslan AA, Toniolo P, Shore RE, Krogh V, Micheli A, Berrino F, Barrett-Connor E, Laughlin GA, Kabuto M, Akiba S, Stevens RG, Neriishi K, Land CE, Cauley JA, Lui LY, Cummings SR, Gunter MJ, Rohan TE, Strickler HD (2011) Circulating sex hormones and breast cancer risk factors in postmenopausal women: reanalysis of 13 studies. Br J Cancer 105(5):709-722

42. Fuhrman BJ, Feigelson HS, Flores R, Gail MH, Xu X, Ravel J, Goedert JJ (2014) Associations of the fecal microbiome with urinary estrogens and estrogen metabolites in postmenopausal women. J Clin Endocrinol Metab 99(12):4632-4640

43. Flores R, Shi J, Fuhrman B, Xu X, Veenstra TD, Gail MH, Gajer P, Ravel J, Goedert JJ (2012) Fecal microbial determinants of fecal and systemic estrogens and estrogen metabolites: a cross-sectional study. J Transl Med 10:253

44. Plottel CS, Blaser MJ (2011) Microbiome and malignancy. Cell Host Microbe 10(4):324-335

45. Chen KL, Madak-Erdogan Z (2016) Estrogen and microbiota crosstalk: should we pay attention? Trends Endocrinol Metab 27(11):752-755

46. Kwa M, Plottel CS, Blaser MJ, Adams S (2016) The intestinal microbiome and estrogen receptor-positive female breast cancer. J Natl Cancer Inst. https://doi.org/10.1093/jnci/djw029

47. Chen KLA, Liu X, Zhao YC, Hieronymi K, Rossi G, Auvil LS, Welge M, Bushell C, Smith RL, Carlson KE, Kim SH, Katzenellenbogen JA, Miller MJ, Madak-Erdogan Z (2018) Long-term administration of conjugated estrogen and bazedoxifene decreased murine fecal beta-glucuronidase activity without impacting overall microbiome community. Sci Rep 8(1):8166

Publisher's Note Springer Nature remains neutral with regard to jurisdictional claims in published maps and institutional affiliations. 